\title{
The first metamagnetic one-dimensional molecular material with nickel(II) and end-to-end azido bridges $\dagger$
}

\author{
Partha Sarathi Mukherjee, ${ }^{a}$ Sudipta Dalai, ${ }^{a}$ Ennio Zangrando, ${ }^{b}$ Francesc Lloret $^{c}$ and Nirmalendu \\ Ray Chaudhuri*a \\ a Department of Inorganic Chemistry, Indian Association for the Cultivation of Science, Jadavpur, \\ Calcutta-700032,India.E-mail:icnrc@mahendra.iacs.res.in \\ ${ }^{b}$ Dipartimento de Scienze Chimiche, University of Trieste, Via Giorgieri 1, 34127 Trieste, Italy \\ ${ }^{c}$ Department de Quimica Inorganica, Universitat de Valencia, Dr. Moliner 50, E-46100 Burjassot, \\ Spain
}

\author{
Received (in Cambridge, UK) 29th May 2001, Accepted 27th June 2001 \\ First published as an Advance Article on the web 19th July 2001
}

\begin{abstract}
A novel single azido bridged one-dimensional Ni(II) chain, which represents the first metamagnetic one-dimensional metal-azido system with only end-to-end azido bridges, has been synthesised and characterised by a low temperature magnetic study.
\end{abstract}

In the last decade chemists have dedicated their efforts to the study of molecular-based magnetic materials owing to the need to understand the fundamental science associated with magnetic interactions between the paramagnetic metal ions and the bridging ligand to develop magneto-structural correlation enabling the designed synthesis of interesting magnetic materials. ${ }^{1}$ One strategy for the design of molecular-based magnets involves assembling of paramagnetic metal ions in one-, twoand three-dimensional networks using suitable bridging ligands. ${ }^{1,2}$ The azido ligand has been widely used because of its diverse binding modes which yield different types of molecules such as dimers, tetramers, one-, two-, and three-dimensional arrays etc. ${ }^{3}$ The prime coordination motif for bridging azido is end-on with ferromagnetic interaction and end-to-end having antiferromagnetic coupling. ${ }^{4}$ To the best of our knowledge all compounds of $\mathrm{Ni}^{\mathrm{II}}$ with end-to-end azido bridges are antiferromagnetic with the exception of two compounds which are weakly ferromagnetic: one is a dimer ${ }^{5}$ and the other a onedimensional chain of $\mathrm{Ni}^{\mathrm{II}}$ using a non-chelating capping ligand. ${ }^{6}$ Examples of metamagnetic molecular materials in the metal-azido system with only end-to-end bridging azido mode are still lacking. Here, we report the synthesis, $\ddagger$ crystal structure and magnetic properties of a new one-dimensional compound, $\left[\mathrm{Ni}(\mathrm{L})\left(\mathrm{N}_{3}\right)_{2}\right]_{n} \mathbf{1}$ ( $\mathrm{L}$ is a tridentate Schiff base obtained by condensation of pyridine-2-aldehyde and N,N,2,2-tetramethylpropane-1,3-diamine) which represents the first example of a metamagnetic molecular material of $\mathrm{Ni}^{\mathrm{II}}$ with only end-to-end azido bridges.

A crystal structure determination§ reveals that complex $\mathbf{1}$ is a single azido bridged one-dimensional $\mathrm{Ni}^{\mathrm{II}}$ chain. A perspective view of the polymeric chain of complex 1 with the atom numbering scheme is shown in Fig. 1. Each nickel atom in the chain has an octahedral coordination environment with a $\mathrm{NiN}_{6}$ core. In the chain, all nickel atoms are linked to one pendant azido in their axial positions. One nitrogen atom, N(7), of the bridging azido is linked to an equatorial position of one nickel while the other nitrogen atom $\mathrm{N}\left(9^{\prime}\right)$ of the same azido is coordinated to an axial position of the neighbouring nickel centre. The equatorial least-square planes of the two $\mathrm{Ni}^{\mathrm{II}}$ centres are not parallel and form a dihedral angle of $31.3(1)^{\circ}$. The deviation of $\mathrm{Ni}^{\mathrm{II}}$ from the best fit square plane towards $\mathrm{N}(4)$ is $0.042(3) \AA$. The two Ni-N-N bond angles of the end-to-end

$\dagger$ Electronic supplementary information (ESI) available: Fig. S1: magnetisation vs. temperature curves at different magnetic fields. Fig. S2: AC susceptibility measurements. See http://www.rsc.org/suppdata/cc/b1/ b104649g/

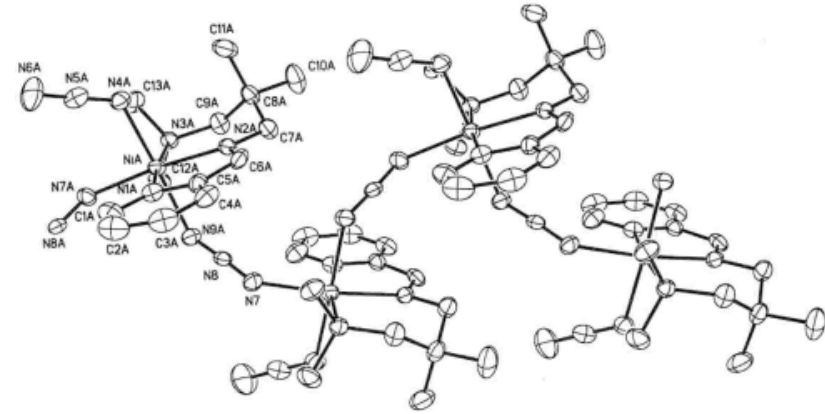

Fig. 1 View of the polymeric chain (40\% thermal ellipsoids) with atom numbering scheme of complex 1 and selected bond lengths $(\AA)$ and angles $\left(^{\circ}\right)$ : Ni-N(1) 2.126(5), Ni-N(2) 2.030(5), Ni-N(3) 2.179(5), Ni-N(4) 2.072(5), Ni-N(7) 2.091(6), Ni-N(9) 2.130(5), N(4)-N(5) 1.172(9), N(5)$\mathrm{N}(6) 1.159(11)$; N(1)-Ni-N(2) 78.5(2), N(1)-Ni-N(3) 172.8(2), N(1)-Ni$\mathrm{N}(7)$ 97.7(2), N(1)-Ni-N(9) 89.8(2), N(2)-Ni-N(9) 85.9(2), N(3)-Ni-N(4) 93.76(19), N(4)-Ni-N(7) 90.3(2), N(4)-N(5)-N(6) 178.4(8).

azido group are different $[\mathrm{N}(8)-\mathrm{N}(7)-\mathrm{Ni} \text { 133.3(4), N(8) })_{-} \mathrm{I}_{-}$ $\mathrm{N}(9)-\mathrm{Ni} 124.5(4)]$. The $\mathrm{Ni}-\mathrm{N}_{3}-\mathrm{Ni}$ torsion angle of $106.8(4)^{\circ}$ is large for an azido $\mathrm{Ni}^{\mathrm{II}}$ compound.6,7 The intrachain distance between adjacent nickel centres is 5.662(1) A.

The thermal variation of $\chi_{\mathrm{M}} T$ is shown in Fig. 2. The nature of the $\chi_{\mathrm{M}} T$ vs. $T$ plot is a signature of global intrachain ferromagnetic interaction. The solid line corresponds to the best fit obtained by considering an uniform ferromagnetically coupled chain of spin triplets (Fig. 2). ${ }^{8}$ The best fitting parameters are $J=+13.5 \mathrm{~cm}^{-1}, g=2.08$ and $R=2.3 \times 10^{-4}$. To the best of our knowledge this is the highest positive $J$ value for end-to-end azido bridged complexes reported to date. 6 Fig. 2 indicates that it is only possible to fit the data from $T>40 \mathrm{~K}$ and below this temperature interchain magnetic interactions are present. In fact, the susceptibility curve shows a maximum at $T$ $=5 \mathrm{~K}$ indicating that an antiferromagnetic ordering is present

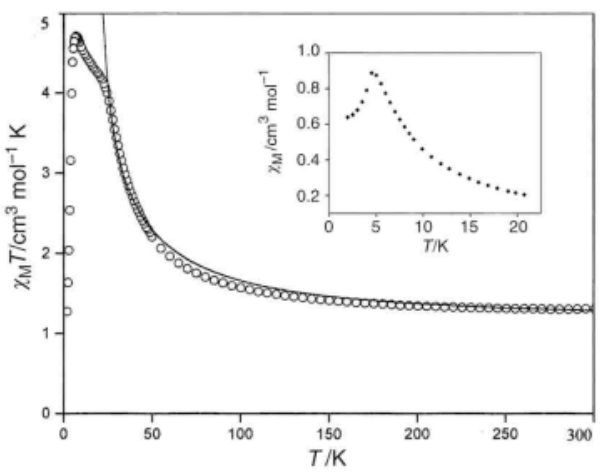

Fig. 2 Plot of $\chi_{\mathrm{M}} T$ vs. $T$ for $\mathbf{1}$. Solid lines show the best fit indicated in the text. Inset: plot of $\chi_{\mathrm{M}} v s . T$ at low temperature. 


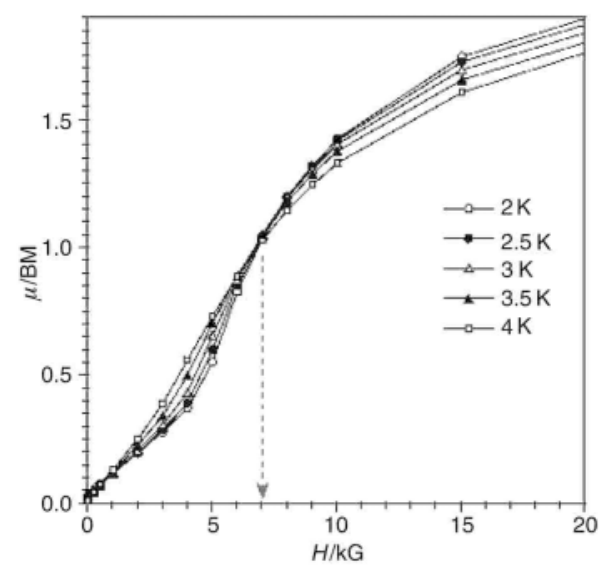

Fig. 3 Magnetization $v s$. applied field curves at different temperatures exhibiting sigmoidal shape with a common crossing point at $H=7 \mathrm{kG}$.

(see inset of Fig. 2). The magnetization curves for different temperatures (Fig. 3) exhibit a sigmoidal shape with a common crossing point for $H=7 \mathrm{kG}$. All these facts clearly indicate a metamagnetic behaviour of the complex with a critical temperature $\left(T_{\mathrm{c}}\right)$ of $5 \mathrm{~K}$ and critical field $\left(H_{\mathrm{c}}\right)$ of $7 \mathrm{kG}$. This magnetic order can be understood by considering that a spin canting structure may be involved, that is the interchain antiferromagnetic coupling does not imply a total antiparallel arrangement of the local spins but that a very weak canted angle should occur. The unusual intrachain ferromagnetic interaction is mainly due to the large deviation of $\mathrm{Ni}-\mathrm{N}-\mathrm{N}$ angles from $108^{\circ}$, the very high $\mathrm{Ni}-\mathrm{N}_{3}-\mathrm{Ni}$ torsion angle and the unusual axial-equatorial bridging mode of the azido. All these structural factors in complex 1 diminish the antiferromagnetic interaction to a greater extent to favour ferromagnetic interaction ${ }^{9}$ as observed. The antiferromagnetic interactions operate between $\mathrm{Ni}^{\mathrm{II}}$ of adjacent chains probably by means of electrostatic (dipolar) interactions between the aromatic ring of the coordinating Schiff base ligand due to a close contact between the parallel aromatic rings from two adjacent chains (the closest distance is $3.41 \AA$ ).${ }^{9}$ When present interchain hydrogen bonding interactions also play a vital role in intermolecular antiferromagnetic interaction. In our system, no classical hydrogen bonds are present, but only $\mathrm{C}-\mathrm{H} \cdots \mathrm{N}$ bonds in which $d(\mathrm{H}-\mathrm{N})$ is ca. $2.5 \AA$ (av.) and $d(\mathrm{C}-\mathrm{N})$ is $3.1 \AA(\mathrm{av}$.), and so in our system the contribution of interchain hydrogen bonding interactions to interchain antiferromagnetic interaction is weak.

Previously reported results show that end-to-end azido bridged metal complexes are always antiferromagnetic. ${ }^{10}$ However, two recently published results show the possibility of ferromagnetic materials with such bridging.5,6 Our present result shows the possibility of a metamagnetic molecular material with only end-to-end azido bridged complexes. The present example is thus a new demonstration of the versatility of azido bridges for building molecular-based magnetic materials. In order to complete the magnetic studies, further experiments along with the DFT calculations and other theoretical calculations are in progress.
We wish to thank the Council of Scientific and Industrial Research, New Delhi for financial support (granted to N. R. C) and Prof. Joan Ribas, Universitat de Barcelona, Spain, for fruitful scientific discussion.

\section{Notes and references}

$\$$ The tridentate Schiff base was prepared by refluxing pyridine-2-aldehyde ( $2 \mathrm{mmol}, 0.214 \mathrm{~g}$ ) and $N, N, 2,2$-tetramethylpropane-1,3-diamine (2 mmol, $0.260 \mathrm{~g})$ in methanol $(10 \mathrm{~mL})$, according to the literature method. ${ }^{11} \mathrm{~A}$ methanolic solution $(10 \mathrm{~mL})$ of nickel(II) perchlorate hexahydrate $(2 \mathrm{mmol}$, $0.730 \mathrm{~g}$ ) was added to the hot methanolic solution $(10 \mathrm{~mL})$ of the tridentate Schiff base ligand $(2 \mathrm{mmol})$. The resulting solution was cooled to room temperature and an aqueous solution $(5 \mathrm{~mL})$ of sodium azide $(4 \mathrm{mmol}, 0.260$ g) was added dropwise with continuous stirring. Suitable green single crystals for structure determination were obtained $(50 \%$ yield) by slow evaporation of this solution. Anal. for $\mathrm{C}_{13} \mathrm{H}_{21} \mathrm{~N}_{9} \mathrm{Ni}: \mathrm{C}, 43.15 ; \mathrm{H}, 5.80 ; \mathrm{N}$, 34.85. Found: C, 43.60; H, 5.65; N, 34.80; IR: $v 2105 \mathrm{~cm}^{-1}$ [ $v_{\mathrm{a}}$ (azide)], $2095 \mathrm{~cm}^{-1}$ (pendant azide).

$\S$ Crystal data for 1: $\mathrm{C}_{13} \mathrm{H}_{21} \mathrm{~N}_{9} \mathrm{Ni}$, monoclinic, space group $P 2{ }_{1} / n, a=$ $11.922(3), b=8.360(2), c=17.121(5) \AA, \beta=101.42(2)^{\circ}, V=1672.6(8)$ $\AA^{3}, Z=4, T=293 \mathrm{~K}, \mu(\mathrm{Mo}-\mathrm{K} \alpha)=1.174 \mathrm{~mm}^{-1}, D_{\mathrm{c}}=1.438 \mathrm{~g} \mathrm{~cm}^{-3}, R 1$ $=0.061$ based on $F, w R 2=0.160$ based on $F^{2}$. Maximum and minimum heights in the final difference Fourier map are 0.910 and -0.360 e $\AA^{-3}$. Intensity data collected on an Enraf-Nonius CAD-4 single crystal diffractometer employing the $\omega-2 \theta$ scan method; absorption correction was applied. The structure was refined using a full matrix refinement procedure (SHELXL 97), with anisotropic thermal parameters assigned to all nonhydrogen atoms. All hydrogen atoms were observed in the difference map.

CCDC reference number 155222. See http://www.rsc.org/suppdata/cc/ b1/b104649g/ for crystallographic data in CIF or other electronic format.

1 J. S. Miller and A. Epstein, Angew. Chem., Int. Ed. Engl., 1994, 33, 385; D. Gatteschi, Adv. Mater., 1994, 6, 635; P. S. Mukherjee, T. K. Maji, G. Mostafa, T. Mallah and N. Ray Chaudhuri, Inorg. Chem., 2000, 39 5147; G. Viau, M. G. Lombardi, G. De Munno, M. Julve, F. Lloret, J. Faus, A. Caneschi and J. M. Clemente-Juan, Chem. Commun., 1997, 1195; C. Ruiz-Perez, J. Sanchiz, M. H. Molina, F. Lloret and M. Julve, Inorg. Chem., 2000, 39, 1363.

2 A. Escuer, R. Vicente, J. Ribas and X. Solans, Inorg. Chem., 1995, 34, 1793; J. Ribas, M. Monfort, C. Diaz, C. Bastos and X. Solans, Inorg. Chem., 1996, 35, 6386.

3 J. Ribas, M. Monfort, R. Costa and X. Solans, Inorg. Chem., 1993, 32, 695; R. Vicente, A. Escuer, J. Ribas, M. S. El Fallah, X. Solans and M. Font-Bardia, Inorg. Chem., 1995, 34, 1278; M. Monfort, J. Ribas and X. Solans, J. Chem. Soc., Chem. Commun., 1993, 350.

4 E. Ruiz, J. Cano, S. Alvarez and P. Alemany, J. Am. Chem. Soc., 1998, 120, 11122.

5 A. Escuer, C. J. Harding, Y. Dussart, J. Nelson, V. Mckee and R. Vicente, J. Chem. Soc., Dalton Trans., 1999, 223.

6 S. Hong and Y. Do, Angew. Chem., Int. Ed., 1999, 38, 193.

7 A. Escuer, R. Vicente, J. Ribas, M. S. El Fallah, X. Solans and M. FontBardia, Inorg. Chem., 1994, 33, 1842.

8 C. Dupas and J. P. Renard, J. Chem. Phys., 1974, 61, 3871.

9 A. Escuer, R. Vicente, J. Ribas, M. S. El Fallah, X. Solans and M. FontBardia, Inorg. Chem., 1993, 32, 3727; N. Matsumoto, Y. Sunatsaki, H. Miyasaka, Y. Hashimoto, D. Luneau and J. P. Tuchagues, Angew. Chem., Int. Ed., 1999, 38, 171.

10 J. Ribas, M. Monfort, C. Diaz, C. Bastos, C. Mer and X. Solans, Inorg. Chem., 1995, 34, 4986.

11 G. Zakrzewski and E. C. Lingafelter, Inorg. Chim. Acta, 1970, 4, 251. 